\title{
ACIDENTES DE TRÂNSITO E VARIÁVEIS PSICOSSOCIAIS - UMA REVISÃO DA LITERATURA
}

\author{
TRAFFIC ACCIDENTS AND PSYCHOSOCIAL VARIABLES - A LITERATURE REVIEW
}

\author{
Sílvia HT Magalhães ${ }^{1}$, Sonia R Loureiro²
}

\begin{abstract}
1'Doutora em Saúde Mental. Responsável Técnica. Serviço de Psicologia. Unidade de Emergência. Hospital das Clínicas da Faculdade de Medicina de Ribeirão Preto - USP. ${ }^{2}$ Docente. Departamento de Neurologia, Psiquiatria e Psicologia Médica. Faculdade de Medicina de Ribeirão Preto - USP

CorRespondência: Dra. Sílvia HT Magalhães. Rua Amália Bissolati Nascimbem, 130 - Residencial Royal Park.

14110-000 - Bonfim Paulista - SP
\end{abstract}

Magalhães SHT, Loureiro SR. Acidentes de trânsito e variáveis psicossociais - Uma revisão da literatura. Medicina (Ribeirão Preto) 2007; 40 (3): 345-51, jul./set.

RESUMO: Objetiva-se identificar e analisar estudos empíricos sobre acidentes de trânsito que abordam variáveis psicossociais como conseqüências ou preditoras do envolvimento com acidentes de trânsito. Procedeu-se ao levantamento bibliográfico eletrônico da literatura indexada, no período de 2000 a 2006, através do PsycINFO, MedLINE e LILACS, utilizando-se as palavraschave: Acidentes de Trânsito, Ansiedade, Depressão, Adaptação Psicossocial e Qualidade de Vida. Foram identificados e analisados 36 artigos agrupados em duas categorias, uma relativa à ansiedade e qualidade de vida pós-acidente e outra relativa às variáveis pessoais dos condutores que se envolveram em acidentes. Os estudos analisados constataram a associação de acidentes de trânsito à ansiedade, depressão, prejuízos psicossociais, identificando como indicadores do envolvimento com os acidentes de trânsito: jovens, sexo masculino, com tendência a agressividade e transgressão social.

Descritotres: Acidentes de Trânsito. Ansiedade. Depressão. Adaptação Psicossocial. Qualidade de Vida.

\section{1- INTRODUÇÃO}

Os acidentes de trânsito (AT) são considerados uma problemática da vida urbana e da civilização suscitada pela popularização dos veículos automotores no cotidiano dos cidadãos. ${ }^{1}$ São referidos pela Organização Mundial de Saúde (OMS) como ocupando mundialmente o $9^{\circ}$ lugar entre as principais causas de mortalidade, representando $2,8 \%$ do total mundial de disfunções e incapacidades. Estima-se que o número de mortes como conseqüência de lesões em AT aumentará de 5,1 milhões em 1990 para 8,4 milhões em $2020 .^{2}$

Em diversos países e inclusive no Brasil os AT são considerados um importante problema de saúde pública pelas implicações, tanto para os indivíduos quanto para a sociedade ${ }^{3}$, em função das seqüelas físicas e psicossociais decorrentes dos acidentes.

Faz-se necessário considerar que as incapacidades decorrentes dos AT podem acometer as pessoas envolvidas, independente de seu papel na determinação da ocorrência do acidente, caracterizandoas como vítimas. O termo vítima de AT é aplicado aos condutores, passageiros ou pedestres, enquanto diferentes atores que têm em comum o envolvimento no evento AT, necessitando de atendimento médico hospitalar, independente da gravidade do evento. ${ }^{2,4,5}$ 
O interesse pelas variáveis psicossociais associadas aos AT se reveste de importância sob duas dimensões, a saber:

a) Como variável conseqüente, enquanto condição de impacto para as vítimas, quanto ao funcionamento psicológico e a qualidade de vida, e

b) como variável antecedente, enquanto características dos condutores que podem favorecer o envolvimento com AT.

A análise dos estudos sob estas duas dimensões pode fornecer subsídios relevantes para orientar práticas reabilitadoras e preventivas.

Neste contexto objetivou-se, com base em uma revisão sistemática da literatura indexada, identificar e analisar estudos empíricos sobre AT que abordam as variáveis psicossociais como conseqüências associadas à dificuldades emocionais e prejuízo na qualidade de vida pós acidente, e como antecedentes ou peculiaridades dos condutores associadas ao envolvimento com AT.

Procedeu-se ao levantamento bibliográfico eletrônico da literatura indexada, no período de 2000 a 2006, nas bases de dados PsycINFO, MedLINE e LILACS utilizando-se as palavras chaves: Traffic Accidents/Acidentes de Trânsito, Anxiety/Ansiedade, Depression/Depressão, Psychosocial Adaptation/ Adaptação Psicossocial e Quality of Life/Qualidade de Vida. Foram incluídos para análise todos os artigos relativos a estudos empíricos, que abordaram variáveis psicológicas relativas a pessoas adultas consideradas vítimas de AT incluindo pedestres, passageiros e/ou condutores. Foram excluídos os artigos que abordaram exclusivamente: os aspectos epidemiológicos dos AT; crianças e adolescentes como vítimas ou condutores; tipos de seqüelas orgânicas decorrentes do AT; modalidades de tratamento do transtorno de stress pós-traumático; uso de álcool e envolvimento com AT e a validação de instrumentos relativos ao comportamento do motorista ao dirigir.

Com base nestes critérios foram identificados 36 artigos e para análise estes foram agrupados em duas categorias, de acordo com os objetivos. Na primeira categoria foram incluídos 16 estudos desenvolvidos com vítimas de AT, abordando a avaliação de preditores do desenvolvimento ou instalação do quadro clínico de transtorno de stress pós-traumático, e os indicadores de prejuízo na qualidade de vida após AT, na segunda, categoria foram incluídos 20 estudos desenvolvidos exclusivamente com condutores, enquanto vítimas de AT, abordando variáveis pessoais e as associações dessas ao envolvimento com AT.

\section{2- PREDITORES DE TRANSTORNO DE STRESS PÓS TRAUMÁTICO (TSPT) E QUALIDADE DE VIDA (QV) PÓS AT}

Do conjunto de 16 artigos incluídos nesta categoria 12 trataram do tema stress pós-traumático e quatro abordaram a temática qualidade de vida.

Os estudos relativos ao TSPT $(\mathrm{N}=12)$, adotaram delineamentos longitudinais comparando o mesmo grupo de participantes em momentos diferentes, em geral o período de um ano foi tomado como referência para a realização das avaliações. Em seis estudos a primeira avaliação foi realizada no período de hospitalização, imediatamente após a ocorrência de $\mathrm{AT}$, ou nas primeiras semanas subseqüentes, não ultrapassando quatro semanas, tal avaliação foi tomada como padrão de comparação com as avaliações posteriores, visando identificar os indicadores com valor preditivo associados ao desenvolvimento de TSPT. As avaliações posteriores foram realizadas aos seis meses e um ano e até três anos após o AT.

As avaliações posteriores, especialmente aos três e seis meses foram realizadas com o objetivo de identificar a instalação do TSPT, tendo em conta que os critérios de diagnóstico para este transtorno estabelecem que ele não deve ser diagnosticado a menos que haja evidência de que surgiu no período de até seis meses após um evento traumático de excepcional gravidade ${ }^{6,7}$, as demais avaliações em geral foram realizadas para confirmar a remissão do TSPT e identificar o valor preditor das variáveis psicológicas presentes nas primeiras avaliações.

Quanto aos estudos sobre QV $(\mathrm{N}=04)$ dois foram longitudinais, sendo que um deles realizou a primeira avaliação durante a hospitalização e a segunda, um ano depois e o outro realizou a primeira avaliação após a hospitalização e a segunda, 24 anos após a ocorrência do AT. Os outros dois estudos adotaram o delineamento transversal, realizando uma única avaliação pós o AT.

Os participantes destes estudos relativos ao TSPT e a QV em geral eram vítimas de AT que necessitaram de hospitalização, identificados durante a internação ou através de registros de arquivos, incluindo condutores, passageiros e pedestres, não fazendo, ao avaliarem os aspectos psicossociais, a distinção destes atores. Em nenhum destes estudos o grau de responsabilidade pessoal pela ocorrência do AT foi abordado não sendo feita referência à culpabilidade como possível fator estressor. 
Como procedimento para a coleta de dados os estudos relativos ao TSPT adotaram avaliações individuais envolvendo a aplicação de escalas padronizadas para a avaliação das variáveis relativas à:

a) TSPT;

b) a gravidade do trauma;

c) a saúde geral; e

d) as características de personalidade, ansiedade e depressão, combinando as medidas obtidas por estes instrumentos aos dados de questionários relativos às variáveis sócio-demográficas.

Os aspectos psicológicos como características de personalidade, ansiedade e depressão, assim como transtornos psiquiátricos prévios, foram considerados como possíveis preditores de desenvolvimento de TSPT. Os estudos avaliaram tais variáveis por meio de inventários de personalidade e de instrumentos padronizados específicos para diagnóstico de sintomas de ansiedade e depressão assim como para diagnóstico de transtorno psiquiátrico prévio.

Os aspectos de QV foram avaliados por meio de questionários gerais e específicos, e de escalas padronizadas.

De modo geral, notou-se uma diversidade de instrumentos utilizados nos estudos que se destinaram a avaliar preditores de QV e de desenvolvimento de TSPT após AT, não se observou uma sugestão explícita de instrumentos que possam ser considerados mais adequados para o estudo das variáveis psicológicas após AT. A Tabela I apresenta os principais resultados relativos ao TSPT e a QV destacados nos artigos analisados.

Um amplo conjunto de variáveis psicológicas foi identificado como preditoras de TSPT, a saber:

a) dissociação persistente;

b) sintomas de ansiedade e depressão;

c) ruminação de idéias, acompanhada de irritabilidade e interpretação negativa da situação.

Os estudos não identificaram um perfil psicológico típico com a presença de variáveis específicas associadas ao desenvolvimento de TSPT destacando uma diversidade de variáveis que podem ser tomadas como preditoras.

A presença de transtornos de personalidade diagnosticados previamente e de quadro psiquiátrico de reação aguda ao stress logo após o AT foram apontados como condições preditoras de TSPT e de possível cronicidade deste quadro ${ }^{17,18,19}$.

Tabela I: Principais resultados - identificação de variáveis preditoras de Transtorno de Stress Pós Traumático (TSPT) (N=12) e de prejuízos para a Qualidade de Vida $(\mathrm{QV})(\mathrm{N}=04)$

Variáveis Preditoras

Estudos*

TSPT $Q V$

\section{Saúde Mental}

1- Reações psicológicas ao stress

- Dissociação

- Ansiedade / Depressão

- Ruminação de idéias

2- Diagnóstico psiquiátrico

- Transtorno de personalidade

- Reação aguda ao stress
$8,9,10,11$

$12,13,14,15$

10,16

17

18,19

Saúde Física

1- Gravidade do trauma

$18,9,16$

20,21

2- Estilo de vida

- $\downarrow$ do convívio social e familiar / impossibilidade de retorno ao trabalho

* Os números correspondem aos artigos ${ }^{8 / 23}$. 
Com relação a saúde física os estudos identificaram a gravidade do trauma como um importante preditor de desenvolvimento de TSPT referindo que, quanto mais grave o trauma maior a probabilidade de desenvolvimento de TSPT, associando o seu desenvolvimento às limitações físicas impostas pelo trauma, principalmente aquelas que dificultam a locomoção. Vale ressaltar que este conjunto de estudos avaliou participantes que, do ponto de vista neurológico, mantinham um razoável estado de consciência. Este critério de inclusão por sua vez circunscreveu os limites da consideração da gravidade do trauma.

Os principais resultados dos estudos relativos ao TSPT apontaram que pessoas que sofreram AT apresentaram prejuízo na adaptação psicossocial após o AT, apresentando sintomas depressivos e de ansiedade que reforçam a sensação de estar doente. Tais manifestações quando associadas à presença de problemas de saúde persistentes dificultaram a retomada das atividades sociais e profissionais. Destacaram ainda como agravante, a pouca disponibilidade de suporte social e de programas de reabilitação chamando a atenção para a necessidade de investimentos dessa natureza na área da saúde.

Nos estudos relativos a QV os principais resultados indicaram que as vítimas de AT em geral apresentaram prejuízos após o evento. Destacaram como condições preditoras de prejuízos na QV pós-acidente: a gravidade do trauma, a presença de sintomas de ansiedade e depressão e a diminuição do convívio social e familiar com dificuldade de retorno ao trabalho. De forma semelhante os estudos relativos ao TSPT destacaram a relevância do suporte social e da reabilitação como condições favorecedoras do retorno ao convívio social e profissional que promovem melhor QV.

\section{3- VARIÁVEIS PESSOAIS DOS CONDUTO- RES E SUAS ASSOCIAÇÕES AOS AT}

Os 20 estudos incluídos nesta categoria adotaram delineamentos transversais, realizando uma única avaliação após o AT tomada como parâmetro para a identificação das variáveis pessoais dos condutores após a ocorrência do AT, sem especificar um período de tempo padrão.

Os participantes foram identificados por meio de consulta a dados de arquivos relativos às notificações policiais de ocorrências de AT ou relativos a registros de atendimento médico hospitalar em decorrência do AT, abrangendo exclusivamente condutores de veículos após o envolvimento em AT.
A coleta de dados, em nove estudos, foi realizada por meio de envio dos instrumentos de avaliação, via correio ou internet para que os participantes respondessem e reenviassem aos pesquisadores. Em geral utilizaram escalas padronizadas específicas para a avaliação das variáveis relativas à:

a) comportamento de dirigir;

b) irritabilidade ao dirigir e

c) características de personalidade, combinando estes instrumentos a questionários gerais de caracterização de variáveis sócio-demográficas e da situação em que o AT ocorreu.

Nos outros onze estudos, o procedimento adotado envolveu a aplicação individual de escalas padronizadas específicas para avaliação dos aspectos de personalidade e do comportamento de dirigir.

A avaliação do comportamento de dirigir foi realizada, em dez estudos, por meio de escalas padronizadas e enfatizou principalmente os erros e transgressões dos condutores de veículos, procurando identificar fatores psicológicos, como agressividade, que poderiam ser considerados como determinantes destes comportamentos. O nível de irritabilidade ao dirigir foi avaliado, em quatro estudos, através de um instrumento padronizado específico para a avaliação desta variável. Os aspectos de personalidade dos condutores foram avaliados em seis estudos por meio de inventários de personalidade diversos. Apesar de utilizarem inventários padronizados que avaliaram a personalidade em aspectos diversos, os estudos focalizaram a análise das variáveis relativas à agressividade.

A Tabela II apresenta os resultados mais relevantes relativos a associação entre variáveis pessoais dos condutores e envolvimento com AT.

Os estudos apontaram que os condutores mais jovens, com idade entre 18 e 20 anos, têm maior probabilidade de envolvimento em AT, justificando que a inexperiência e a tendência a atribuir um caráter de diversão ao ato de dirigir, fazem com que tenham pouca consciência das conseqüências de seu comportamento no trânsito. Os homens foram considerados mais propensos ao envolvimento em AT, em alguns estudos este risco foi duas vezes maior do que o das mulheres $27,28,30,31$.

A agressividade e a irritabilidade ao dirigir foram também consideradas como condições preditoras. Os estudos identificaram que condutores mais agressivos e mais irritáveis ao dirigir têm maior probabilidade de envolvimento com AT. A agressividade foi defi- 
Tabela II: Principais resultados - Identificação das características dos condutores relacionadas ao envolvimento em Acidentes de Trânsito (AT) (N=14)

\begin{tabular}{lll}
\hline Objetivo $/ \boldsymbol{N}^{\circ}$ de estudos & Variáveis Preditoras & Estudos $^{*}$ \\
\hline Características dos condutores & Aspectos demográficos & \\
(14) & - Idade $-18 / 20$ anos & $24,25,26,27,28$ \\
& - Gênero - Masculino & $29,30,27,31,32,33$ \\
& Aspectos psicológicos e o & \\
& comportamento de dirigir & \\
& - Irritabilidade / Intolerância & $34,29,35,36,37,38,39,40,41,42$ \\
& - Agressividade / Transgressão & $30,38,40,41,43$ \\
\hline
\end{tabular}

* Os números correspondem aos artigos ${ }^{24 / 43}$.

nida como uma tendência ao desrespeito às normas de trânsito vigentes, fazendo uso do veículo como uma forma de agredir a si e à sociedade. A irritabilidade foi definida como pouca tolerância e hostilidade diante das características rotineiras de organização do trânsito, sendo este caracterizado como um importante fator estressor para o condutor.

Alguns estudos apontaram para a necessidade de se identificar se as características de agressividade e irritabilidade são indicadores mais estáveis e estruturais da personalidade do condutor ou se estão relacionadas a aspectos mais reativos e funcionais, caracterizando variáveis situacionais.

A tendência a transgredir as normas de trânsito foi identificada como um indicador de agressividade ao dirigir, facilitando o envolvimento com AT, constatando que os condutores com tendências a não respeitar os índices de velocidade, a não utilizar cinto de segurança e a não respeitar a sinalização se envolvem mais com AT.

Nos estudos analisados observou-se uma diversidade de variáveis psicossociais focalizadas e pouca clareza quanto ao período de tempo pós AT tomado como referência, o que dificultou as comparações.

Ressalta-se, ainda, que a explicitação da culpabilidade pelo AT pode ser uma variável que interfere na avaliação do próprio comportamento feita pelos envolvidos. Tal explicitação é complexa por envolver não só as vítimas como as condições de contexto e de regulamentação do trânsito além das leis que regem a responsabilidade civil dos cidadãos com suas peculiaridades em diferentes países ${ }^{44}$.
Em todos os estudos analisados as variáveis preditoras associadas ao envolvimento com AT identificadas em amostras com história de envolvimento com AT o que de certo modo limita o alcance dos dados no que diz respeito ao seu valor preditor para condutores em geral, independentemente do envolvimento prévio com AT.

\section{4- CONSIDERAÇÕES FINAIS}

De um modo geral, os estudos que objetivaram identificar as conseqüências dos AT na adaptação psicossocial e para a QV dos envolvidos, constataram o impacto negativo para o funcionamento psicológico das vítimas. Quanto às características preditoras do possível envolvimento com AT verificaram que as condições como idade jovem, sexo masculino e a tendência a agressividade e transgressão social foram as mais associadas ao risco, caracterizando indicações específicas para programas de prevenção em saúde.

Os estudos analisados foram conduzidos em quatro países diferentes e não fizeram referência ao contexto social e de regulamentação do trânsito nestes locais como variável incluída na análise, possivelmente não dando destaque a interferência destes aspectos situacionais na ocorrência do AT. Focalizaram a descrição de um perfil psicológico, buscando identificar características pessoais preditoras do envolvimento em AT, sem destaque para as variáveis ambientais. Neste sentido, os delineamentos adotados nesses estudos se contrapõem às considerações que apontam que o trânsito deve ser considerado como um pro- 
blema social onde os antecedentes causadores dos eventos não devam se restringir ao estudo da subjetividade do participante, mas deve abranger a sua inserção num contexto ambiental complexo, que considere o impacto do seu comportamento sobre o ambiente e do ambiente sobre seu comportamento. ${ }^{45}$

Nos estudos analisados as variáveis pessoais do condutor foram tomadas como as principais condições preditoras de envolvimento em AT. Consideramse necessários novos estudos que visem a identificação dos processos associados ao comportamento de dirigir, considerando as características psicológicas individuais de forma mais ampla, assim como as condições ambientais envolvendo a regulamentação e organização do trânsito.

Outro ponto a ser destacado diz respeito à ausência de referência a aspectos de culpabilidade dos envolvidos e suas implicações enquanto um fator estressor relevante que pode interferir na recupera- ção pós AT. Tal variável é complexa por envolver questões de natureza diversa como a legal envolvendo a prescrição de normas e punições e a pessoal, de fórum íntimo, que envolve o sentido de responsabilidade e atribuição de causalidade.

Ao analisar os estudos empíricos recentes identificados por meio de pesquisa sistemática nas bases indexadas, constata-se a relevância das variáveis psicossociais enquanto uma medida das conseqüências dos AT para as vítimas e como um recurso de avaliação das características pessoais dos condutores que podem funcionar como condições de risco favoráveis ao envolvimento com AT. Na área da saúde o conhecimento de tais dimensões pode instrumentar práticas preventivas e de reabilitação. Destaca-se como relevante que os aspectos psicossociais sejam levados em conta nos programas de reabilitação pós-trauma para as vítimas de AT, dadas as especificidades dessa população.

Magalhães SHT, Loureiro SR. Traffic accidents and psychosocial variables - A literature review. Medicina (Ribeirão Preto) 2007; 40 (3): 345-51, july/sept.

ABSTRACT: The objective of this study was to identify and analyze empirical studies on traffic accidents in terms of psychosocial variables as consequences or predictors of involvement in traffic accidents. An electronic bibliographic search of literature indexed with PsycINFO, MedLINE and LILACS, between 2000 to 2006, was undertaken using the key words: Traffic Accidents, Anxiety, Depression, Psychosocial Adaptation and Quality of Life. A total of 36 articles were identified and grouped into two categories, one relative to anxiety and quality of life after accident and the other relative to the personal variables of the drivers. The studies evaluated associated traffic accidents with anxiety and depression, psychosocial loss, identifying the following characteristics as indicators of involvement in traffic accidents: young, male, with tendency to aggressiveness and social transgression.

Keywords: Accidents, Traffic. Anxiety. Depression. Psychosocial Adaptation. Quality of Life.

\section{REFERÊNCIAS}

1 - Ott EA, Favaretto AL, Neto AFPR, Zechin JG, Bordin R. Acidentes de trânsito em área metropolitana da região Sul do Brasil - Caracterização da vítima e das lesões. Rev Saúde Pública 2003; 27 (5): 350-6.

2 - Hoffmann MH, Gonzáles AD. Acidentes de trânsito e fator humano. In: M.H. Hoffmann, R.M. Cruz, J.C. Alchieri, orgs. Comportamento humano no trânsito. São Paulo: Casa do Psicólogo; 2003. p. 375-92.

3 - Marín-Léon L, Vozzotti MM. Comportamentos no trânsito: um estudo epidemiológico com estudantes universitários. Cad Saúde Pública 2003; 19(2):515-23.

4 - Brysiewicz P. Pedestrian road traffic collisions in South Africa. Accid Emerg Nurs 2001; 9 (3):194-7.
5 - Marin L, Queiroz MS. A atualidade dos acidentes de trânsito na era da velocidade: uma visão geral. Cad Saúde Pública 2001; 16 (1): 59-68.

6 - Organização Mundial da Saúde. Classificação dos transtornos mentais e de comportamento da CID-10: Descrições clínicas e diretrizes diagnósticas. Porto Alegre: Artes Médicas; 1993.

7 - American Psychiatric Association. Diagnostic and Statistical Manual of Mental Disorders. 4th. ed. Washington, DC: APA; 1994.

8 - Dougal A, Ursano RJ, Posluszny DM, Fullerton CS, Baum A. Predicors of posttraumatic stress among victims of motor vehicle accidents. Psychosom Med 2001; 63(3): 404-11.

9 - Friedland JF, Dawson DR. Function after motor vehicle accidents: a prospective study of mild head injury and posttraumatic stress, J Nerv Ment Dis 2001, 189(7): 426-34. 
10 - Holeva V, Tarrier N. Personality and peritraumatic dissociation in the prediction of PTSD in victims of road traffic accidents. J Psychosom Res 2001; 51(5): 687-92.

11 - Murray J, Ehlers A, Mayou RA. Dissociation and posttraumatic stress disorder: Two prospective studies of road traffic accident survivors. Br J Psychiatry 2002; 180 (4): 363-8.

12 - Mayou RA, Ehlers A, Bryant B. Posttraumatic stress disorder after motor vehicle accidents: 3 year follow-up of a prospective longitudinal study. Behav Res Ther 2002; 40 (6): 665-75.

13 - Mayou R, Bryant B. Outcome in consecutive emergency department attenders following a road traffic accident. $\mathrm{Br} \mathrm{J}$ Psychiatry 2001;179: 528-34.

14 - Mayou R, Bryant B, Ehlers A. Predction of psychological outcomes one year after a motor vehicle accident. Am J Psychiatry 2001; 158(8): 1231-8.

15 - Mayou RA, Bryant B. Outcome 3 years after a road traffic accident. Psychol Med 2002; 32 (4): 671-5.

16 - Jeavons S. Predcting who suffers psychological trauma in the first year after a road accident. Behav Res Ther 2000; 38(5): 499-508.

17 - Malta LS, Blanchard EB, Taylor AE, Hickling EJ, Freidenberg BM. Personality disorders and posttraumatic stress disorder in motor vehicle accident survivors. J Nerv Ment Dis 2002; 190(11): 767-74.

18 - Bryant PA, Harvey AG, Guthrie RM, Moulds ML. A prospective study of psychophysiological arousal, acute stress disorder and posttraumatic stress disorder. J Abnorm Psychol 2000; 109(2): 341-4.

19 - Lucas JL. Driver's psychological and physical reactions after motor vehicle accidents. Transp Res Part F: Traffic Psychol Behav 2003; 6: 135-45.

20 - Peltzer K, Renner W. Psychosocial correlates of the impact of road traffic accidents among South African drivers and passangers. Accid Anal Prev 2004; 36: 367-74.

21 - Ho R, Davidson G, Van-Dyke M, Agar-Wilson M. The impact of motor vehicle accidents on the psychological well-being of at-fault drivers and related passengers. J Health Psychol 2000; 5(1): 33-51.

22 - Pirente N, Gregor A, Bouillon B, Neugebauer E. Quality of life of severely injured patientes 1 year after trauma. A matched pair study compared with a health control group. Unfallchirurg 2001; 104(1):57-63.

23 - Steadman-Pare D, Colantonio A, Ratcliff G, Svernich L. Factors associated with perceived quality of life many Years after traumatic brain injury. J Head Trauma Rehabil 2001; 16(4):330-42.

24 - Akerstedt T, Kecklund G. Age, gender andearly morning highway accidents. J Sleep Res 2001; 10(2): 105-10.

25 - Al-Madani H, Al-Janahi AR. Assessment of drivers comprehension of traffic signs based on their traffic, personal, and social characteristics. Transp Res Part F: Traffic Psychol Behav 2002; 5(1): 63-76.

26 - Ballesteros MF, Dischinger PC. Characteristics of traffic crashes in Mayland (1996-1998): diferences among youngest drivers. Accid Anal Prev 2002; 34 (3): 279-84.

27 - Lam LT. Distractions and the risk of car crash injury: the effect of driver's age. J Safety Res 2002; 33 (3): 411-9.

28 - El Chliaoutakis J, Demakokos P, Tzamalouka G, Bakou V, Koumaki M, Darviri C. Agressive behavior while driving as predictor of self-reported car crashes. J Safety Res 2002; 33(4): 431-43.
29 - Bastos YGL, Andrade SM, Soares DA. Características dos acidentes de trânsito e das vítimas atendidas em serviço pré hospitalar em cidade do sul do Brasil, 1997/2000. Cad Saúde Pública 2005; 21(3): 815-22.

30 - Karlaftis MG, Kotzampassakis I, Kanellaidis G. An empirical investigation of European driver's self assessment. J Safety Res 2003; 34(2): 207-13.

31 - Tavris DR, Kuhn EM, Layde PM. Age and gender patterns in motor vehicle crash injuries: importance of type crash and accupant role. Accid Anal Prev 2001; 33(2): 167-72.

32 - Worrel SS, Koepsell TD, Sabath DR, Gentilello LM, Mock CN, Nathens $A B$. The risk reinjury in relation to tima since first injury: a retrospective population based study. J Trauma 2006; 60(2): 379-84.

33 - Hasselberg M, Laflamme L. The social patterning of injury repetitions among young car drivers in Sweden. Accid Anal Prev 2005; 37(1):163-8.

34 - Arthur WJ, Doverspike D. Predicting motor vehicle crash involvement of a personality measure and drivin knowledge test. J Prev Interv Community 2001; 22(1): 35-42.

35 - Iversen $\mathrm{H}$, Rundmo T. Personality risky driving and accident involvement among Norwegian drivers. Pers Individ Dif 2002; 5(1): 63-76.

36 - Levine B, Dawson D, Boutet I, Schwartz ML, Stuss D. Assessment of strategic self-regulation in traumatic brain injury: its Relationship to injury severity and psychosocial outcome. Neuropsychology 2000; 14(4):191-200.

37 - Mesken J, Lajunen T, Summala $\mathrm{H}$. Interpersonal violations, speeding violations and their retion to accident involvement in Finland. Ergonomics 2002; 45(7): 469-83.

38 - Nochi M. Reconstructing self-narratives in coping with traumatic brain injury. Soc Sci Med 2000; 51(12):1795-804.

39 - Parker D, Lajunen TC, Summala H. Anger and agression among drivers in three European countries. Accid Anal Prev 2002; 34: 229-35.

40 - Ullerberg P. Personality subtypes of young drivers. Relationship to risk - taking preferences, accident envolvement and responses to traffic safety campaing, Transp Res Part F: Traffic Psychol Behav 2001; 4 (4): 279-97.

41 - Dahlen ER, Martin RC, Ragan K, Kuhlman MM. Driving anger, sensation seeking, impulsiveness, and boredom proneness in the prediction of unsafe driving. Accid Anal Prev 2005; 37: 341-8.

42 - Sümer N. Personality and behavioral predictors of traffic accidents: testing a contextual mediated model. Accid Anal Prev 2003 ; 35(6): 949-64.

43 - Shinar D, Compton R. Aggressive driving: an observational study of driver, vehicle, and situational variables. Accid Anal Prev 2004; 36(3): 429-37.

44 - Wahlberg AE. Some methodological deficiences in studies on traffic accidents predictors. Accid Anal Prev 2003; 35(4): 473-86.

45 - Günter H Ambiente, Psicologia e trânsito: reflexões sobre uma integração necessária. In: Hoffmann MH, Cruz RM, Alchieri JC, orgs. Comportamento humano no trânsito. São Paulo: Casa do Psicólogo; 2003. p.47-57.

Recebido em 27/06/2007

Aprovado em 21/09/2007 